Gustavo Adolfo Lanza Bayona

\title{
Desenvolvimento de filmes de ITO para dispositivos orgânicos eletroluminescentes invertidos (IOLEDs) e transparentes (TOLEDs)
}

Dissertação apresentada como requisito parcial para obtenção do grau de Mestre pelo Programa de Pós-graduação em Física do Departamento de Física da PUC-Rio

Orientador: Prof. Marco Cremona 


\title{
Gustavo Adolfo Lanza Bayona
}

\section{Desenvolvimento de filmes de ITO para dispositivos orgânicos eletroluminescentes invertidos (IOLEDs) e transparentes (TOLEDs)}

Dissertação apresentada como requisito parcial para obtenção do grau de Mestre pelo Programa de Pós-graduação em Física do Departamento de Física do Centro Técnico Científico da PUCRio. Aprovada pela Comissão Examinadora abaixo assinada.

\author{
Prof. Marco Cremona \\ Orientador \\ Departamento de Física - PUC-Rio \\ Prof. Marcelo Eduardo Huguenin Maia da Costa \\ Departamento de Física - PUC-Rio
}

Prof. Cristiano Legnani

UFJF

Prof. José Eugênio Leal

Coordenador Setorial do Centro Técnico Científico - PUC-Rio 
Todos os direitos reservados. É proibida a reprodução total ou parcial do trabalho sem autorização da universidade, do autor e do orientador.

\section{Gustavo Adolfo Lanza Bayona}

Graduado em Licenciatura em Física pela Universidad de Cundinamarca (Fggá, Colômbia-2009). Tem experiência na área de Física da Matéria Condensada, com ênfase em filmes finos de óxidos condutores transparentes e dispositivos orgânicos eletroluminescentes (OLEDs).

Ficha Catalográfica

Lanza, Gustavo

Desenvolvimento

de

filmes de ITO para dispositivos orgânicos eletroluminescentes invertidos (IOLEDs) e transparentes (TOLEDs) / Gustavo Adolfo Lanza Bayona; orientador: Marco Cremona. - Rio de Janeiro : PUC-Rio, Departamento de Física, 2012.

v., 106 f.: il. ; $30 \mathrm{~cm}$

1. Dissertação (mestrado) - Pontifícia Universidade Católica do Rio de Janeiro, Departamento de Física.

Inclui referências bibliográficas.

1. Física - Tese. 2. Óxidos condutores transparentes. 3. ITO. 4. Filmes finos. 5. TOLED. 6. IOLED. 7. pulverização catódica. I; Cremona, Marco; II; Pontifícia Universidade Católica do Rio de Janeiro. Departamento de Física; III; Título; 
para Valentina e Karen minhas belas princesas 


\section{Agradecimentos}

Agradeço a Deus por tudo o que ele me dá.

A minha filha Valentina e à minha esposa Karen, pelo amor, paciência e apoio incondicionais ao longo destes anos.

A meus pais Martha e Edgar, por acreditar em mim e em minhas decisões, pelo amor, esforço e dedicação para que eu sempre seja feliz.

Ao meu orientador, Marco Cremona, pela orientação neste trabalho, por ter me proporcionado um trabalho novo, empolgante e bonito, pela confiança, ensinamentos e reconhecimento.

Aos meus amigos Harold e Juan, pela amizade sincera, convivência, paciência e ajuda nos momentos difíceis. Obrigado por ser minha família em Rio.

Aos colegas do LOEM, Yolanda, Kelly, Narely, Bia e em especial á Vanessa e ao Rafael, meus professores de português. As suas contribuições foram fundamentais para a realização deste trabalho.

Aos "majes".

Aos amigos da favelinha, Alex, Jazmine e Johana, pelas risadas e conversas.

Aos funcionários do Depto. de Física da PUC-Rio, pela ajuda, sempre com enorme competência.

A todos aqueles que de uma forma ou de outra contribuíram para que este trabalho fosse concluído.

À CAPES, CNPq, RENAMI (Rede de Nanotecnologia Molecular e de Interfaces) e FAPERJ pelo apoio financeiro, e à PUC-Rio, pela utilização das instalações e laboratórios. 


\section{Resumo}

Lanza, Gustavo; Cremona, Marco. Desenvolvimento de filmes de ITO para dispositivos orgânicos eletroluminescentes invertidos (IOLEDs) e transparentes (TOLEDs). Rio de Janeiro, 2012. 106p. Dissertação de Mestrado - Departamento de Física, Pontifícia Universidade Católica do Rio de Janeiro.

Neste trabalho são apresentados os resultados da produção e caracterização de dispositivos orgânicos emissores de luz invertidos (IOLEDs) e transparentes (TOLEDs). Como eletrodo superior transparente, utilizouse o óxido de índio estanho (ITO), que foi depositado via pulverização catódica assistida por radiofreqüência sobre camadas protetoras orgânicas (CuPC) e metálicas (Alumínio). Para evitar possíveis danos efetuados nas camadas do dispositivo pelo processo de pulverização catódica, as deposições de ITO foram realizadas a baixa potência. Primeiramente, os filmes de ITO foram caracterizados elétrica e opticamente. A seguir, foi estudada a interação entre a superfície das camadas protetoras (CuPC e Alumínio) e o filme de ITO. Por fim, os dispositivos IOLEDs e TOLEDs foram caracterizados através de medidas de eletroluminescência, densidade de corrente e luminância, todas elas, em função da tensão aplicada. A partir destes estudos foi possível produzir dispositivos IOLEDs sobre substratos opacos além de dispositivos TOLEDs com transmitância média de $70 \%$ na região do espectro visível.

\section{Palavras-chave}

Óxidos condutores transparentes; ITO; Filmes finos; TOLED; IOLED; pulverização catódica; 


\section{Abstract}

Lanza, Gustavo; Cremona, Marco (Advisor). Development of ITO thin films for inverted (IOLEDs) and transparent (TOLEDs) organic electroluminescent devices. Rio de Janeiro, 2012. 106p. MSc Dissertation — Departamento de Física, Pontifícia Universidade Católica do Rio de Janeiro.

This work presents the results of production and characterization of organic light emitting devices inverted (IOLEDs) and transparent (TOLEDs). As transparent top electrode, a thin film of indium tin oxide (ITO) deposited via rf magnetron sputtering was used. The ITO films were deposited onto organic ( $\mathrm{CuPC}$ ) and metal (Aluminum) protective layers. In order to prevent the damage incurred on the organic and metal layers by the sputtering process, the ITO deposition is carried out at room temperature and under low rf power. First, the ITO films were characterized by electrical and optical measures. Next, the interaction between the surface of the protective layers ( $\mathrm{CuPc}$ and Aluminum) and the ITO film was analyzed. Finally, the IOLEDs and TOLEDs devices were characterized by electroluminescence, current density and luminance measures, all as a function of the applied voltage. From these studies, was possible to produce IOLEDs devices onto opaque substrates and TOLEDs devices whit average transmittance of $70 \%$ in the visible range.

\section{Keywords}

Transparent Conductive Oxides; ITO; Thin Films; TOLED; IOLED; IOLED; 


\section{Sumário}

1 Introdução 15

1.1 Desenvolvimento de um cátodo transparente 18

$\begin{array}{ll}1.2 \text { Justificativa } & 19\end{array}$

$\begin{array}{ll}1.3 \text { Objetivos } & 20\end{array}$

$\begin{array}{lll}\text { 1.3.1 Objetivos espeficos } & 20\end{array}$

$\begin{array}{lll}1.4 & \text { Revisão Bibliográfica } & 21\end{array}$

2 Aspectos Teóricos $\quad \mathbf{2 6}$

2.1 Dispositivos Orgânicos Emissores de Luz - OLEDs 26

2.1.1 Semicondutores Orgânicos 26

2.1.2 Arquitetura e fabricação de OLEDs $\quad 27$

2.1.3 Funcionamento 28

$\begin{array}{ll}2.1 .4 & \text { Injeção } \\ 2.5 & 29\end{array}$

2.1.5 Eletroluminescência 31

2.1.6 Materiais orgânicos 32

2.1.7 Eletrodos 32

2.2 Oxido de índio dopado com estanho 34

2.2.1 Propriedades dos filmes de oxido de índio dopado com estanho 34

2.2.2 ITO depositado por R. F. magnetron sputtering 36

2.3 ITO como eletrodo superior em Dispositivos Orgânicos Emissores de Luz $\quad 40$

2.3.1 Dispositivos Orgânicos Emissores de Luz Invertidos - IOLEDs 42

2.3.2 Dispositivos Orgânicos Emissores de Luz Transparentes - TOLEDs 43

3 Procedimentos Experimentais $\quad 45$

3.1 Materiais orgânicos $\quad 45$

3.2 Substratos 46

3.3 Produção de filmes finos 47

3.3.1 Deposição física de filmes finos por evaporação térmica resistiva 48

3.3.2 R. F. Magnetron Sputtering 50

3.4 Fabricação dos dispositivos $\quad 51$

3.4.1 Parâmetros utilizados na produção dos filmes finos e dos dispositivos 52

3.4.2 Estrutura dos dispositivos 53

3.4.3 Estrutura IOLED 53

$\begin{array}{ll}3.4 .4 & \text { Estrutura TOLED }\end{array}$

3.5 Métodos e equipamentos de análise $\quad 55$

3.5.1 Perfilometria $\quad 55$

3.5.2 Medida do efeito Hall $\quad 56$

3.5.3 Espectroscopia óptica 58

3.5.4 Microscopia de força atômica (AFM) 59

3.5.5 Análise de Feixe de Íons Focalizados (FIB) 59

3.5.6 Eletroluminescência $\quad 59$ 
3.5.7 Medidas de Corrente e Potência Luminosa em OLEDs 61

4 Resultados e discussão $\quad 62$

4.1 Caracterização dos filmes finos de ITO 62

4.1.1 Caracterização Elétrica 64

4.1.2 Caracterização óptica 66

$\begin{array}{lll}4.2 & \text { IOLEDs } & 68\end{array}$

$\begin{array}{lll}4.2 .1 & \text { Interface CuPC/ITO } & 68\end{array}$

$\begin{array}{ll}\text { 4.2.2 Caracterização dos dispositivos } & 71\end{array}$

4.3 TOLEDs 84

4.3.1 Filmes finos de Al 84

4.3.2 Caracterização dos dispositivos $\quad 88$

$\begin{array}{llr}5 & \text { Conclusões e perspectivas } & 96\end{array}$ 


\section{Lista de figuras}

1.1 Emissão de luz em um dispositivo invertido (IOLED) e em um dispositivo transparente (TOLED).

1.2 IOLEDs e TOLEDs comerciais. a. TV Super Top Emission da Sony, b Celular Xperia Pureness da Sony Ericsson, c. mp3 IceTouch YPH1 e d.Dispositivos de sinalização da Novaled.

1.3 Esquema do possível dano devido à deposição de um filme de ITO sobre camadas orgânicas.

2.1 Arquiteturas dos OLEDs, a. bicamada. b. multicamada. Onde CIB é camada injetora de buracos, CTB é camada transportadora de buracos, CE é camada emissora, CTE é camada transportadora de elétrons e CIE é camada injetora de elétrons.

2.2 Processo de eletroluminescência para um dispositivo bicamada. 29

2.3 Vacância aniônica numa estrutura tipo bixbyite. 34

2.4 Bandas de energia do óxido de índio dopado com estanho 35

2.5 Esquema dos efeitos de partículas energéticas bombardeando uma superfície

2.6 Representação esquemática do R. F. Magnetron Sputtering. 39

2.7 Interface metal semicondutor.

2.8 Arquiteturas para um IOLED. Onde CIB é camada injetora de buracos, CTB é camada transportadora de buracos, CE é camada eletroluminescente.

2.9 Arquiteturas para um TOLED. Onde CTB é camada transportadora de buracos, CE é camada eletroluminescente e CIE é camada injetora de elétrons.

3.1 Fórmulas estruturais dos materiais utilizados neste trabalho.

3.2 Obtenção dos padrões geométricos de ITO através do proceso de litografia.

3.3 Sistema para a deposição de filmes finos UNIVEX 300

3.4 Sistema utilizado na fabricação de filmes finos de ITO: UNIVEX 450
3.5 Estrutura do IOLED. (a) Substrato com padrão de ITO(comercial), (b) Deposição do Al, (c) Deposição materiais orgânicos e (d) Deposição ITO.

3.6 Estrutura do TOLED. (a) Substrato com padrão de ITO(comercial), (b) Deposição materiais orgânicos, (c) Deposição filme fino de Al e (d) Deposição ITO.

3.7 Perfilômetro Veeco Dektak 150.

3.8 Montagem experimental para a realização de medidas de resistividade e efeito Hall através do método de Van der Pauw

3.9 Equipamento de medida e porta amostra para medidas de efeito Hall da ECOPIA Hall Effect Measurement System HS 3000. 
3.10 Esquema do espectrofluorímetro da Photon Technology International (PTI) Quanta Master ${ }^{\mathrm{TM}} 30$ utilizado para aquisição dos espectros de eletroluminescência.

3.11 Acessório implementado para a realização das medidas de eletroluminescência.

4.1 Taxa e tempo de deposição em função da potência, para filmes de ITO de $100 \pm 5 \mathrm{~nm}$.

4.2 Propriedades elétricas: número de portadores $(n)$, mobilidade dos portadores $(\mu)$ e resistência de folha $\mathrm{R}_{\square}$ dos filmes de ITO para as diferentes potências de rf utilizadas.

4.3 Triângulos vermelhos: valores da resistência de folha com seus respectivos erros. Linha preta: ajuste do tempo de deposição em função da potência.

4.4 Espectros de transmitância para os filmes de ITO depositados a diferentes potências. Grafico inserido: resultados da transmitância obtidos para um comprimento de onda de $550 \mathrm{~nm}$.

4.5 Coeficiente de absorção óptica ao quadrado em função da energia para o cálculo do valor da banda proibida dos filmes de ITO.

4.6 Imagens topográficas de AFM dos filmes de CuPC sobre Si, ITO sobre Si e ITO depositado sobre CuPc.

4.7 Imagens FIB da seção transversal de uma bicamada CuPC/ITO depositada Si.

4.8 Fotografia de um IOLED em funcionamento. Os pontos pretos são gerados pela colisão dos íon e átomos com as moléculas do filme orgânico.

4.9 Espectro de eletroluminescência de IOLEDs fabricados com ITO depositado a diferentes potências.

4.10 Espectro de eletroluminescência (EL) do $\mathrm{Alq}_{3}$ em IOLEDs. Inseridas: fotografias do dispositivo fabricado com ITO depositado a 8 e $20 \mathrm{~W}$.

4.11 Intensidades dos picos de EL para diferentes tensões de operação. $\quad 74$

4.12 Parâmetro $\beta$ (equação(4.1)) em função da potência de deposição. $\quad 75$

4.13 Espectro de eletroluminescência de um IOLED fabricado com ITO depositado a $40 \mathrm{~W}$ para diferentes tensões de operação.

4.14 Curva J-V para IOLEDs fabricados com ITO depositado a diferentes potências.

4.15 Característica L-V para IOLEDs fabricados com ITO depositado a diferentes potências. Inserido: curvas J-V e L-V para o IOLED com ITO depositado a $14 \mathrm{~W}$.

4.16 Característica J-V em escala logarítmica para estudo das propriedades de transporte dos IOLEDs.

4.17 Comparação das característica J-V entre o IOLED e outros dispositivos. Inserido: Comparação da EL entre um IOLED e um OLED baseados em $\mathrm{Alq}_{3}$. 
4.18 Ajuste do modelo de injeção por tunelamento $\left(\frac{1}{V}\right.$ vs. $\left.\log \left(\frac{J}{V^{2}}\right)\right)$ para as características elétricas de um IOLED, OLED com ITO depositado a $20 \mathrm{~W}$ e um OLED com ITO comercial. As duas regiões determinam a predominância da carga injetada ( $n$ ou $p$ )

4.19 Esquerda: curva I-V e EL de um IOLED crescido sobre Si, direita: fotografia do dispositivo em operação.

4.20 Fotografias de um IOLED crescido sobre papel. Da esquerda para direita: 1. dispositivo desligado, 2. dispositivo ligado e 3. dispositivo quando inicia a queimar-se.

4.21 Imagens topográficas AFM de filmes de Al de 5, 8 e 15nm. $\quad 85$

4.22 Imagens topográficas de AFM dos filmes.

4.23 Imagens MEV de filmes de Al de 5 nm (a) e 10 nm (b). $\quad 87$

4.24 Transmitância de um filme de Al em função de sua espessura. $\quad 87$

4.25 Processo de "cura" sofrido pelos TOLEDs.

4.26 Transmitância de TOLEDs com espessura (t) para o Al de 5, 7 e $10 \pm 1 \mathrm{~nm}$.

4.27 Espectro de eletroluminescência (EL) do $\mathrm{Alq}_{3}$ para TOLEDs com diferente espessura de Al.

4.28 Característica J-V em escala logarítmica para estudo das propriedades de transporte dos TOLEDs.

4.29 Comparação das característica L-V entre um OLED e TOLEDs com diferente espessura para o Al.

4.30 Ajuste do modelo de injeção por tunelamento $\left(\frac{1}{V}\right.$ vs. $\left.\log \left(\frac{j}{V^{2}}\right)\right)$ para as características elétricas de um OLED e TOLEDs com diferente espessura para o Al. As duas regiões determinam a predominância da carga injetada ( $n$ ou $p$ ).

4.31 Fotografias de TOLEDs em funcionamento. 


\section{Lista de tabelas}

1.1 Características de IOLEDs e TOLEDs encontradas na literatura. 25

3.1 Nomes e fórmulas dos compostos utilizados neste trabalho 45

3.2 Parâmetros de calibração do sistema INFICOM para controle da espessura e das taxas de deposição. 50

4.1 Densidades de potência utilizadas neste trabalho. 63

4.2 Características da deposição dos filmes de Al em estudo, onde T é a taxa de deposição e t é a espessura. 
Nunca deixe o medo de errar impedir que você jogue 